\title{
M. Maurizio, V.S. Tomelleri (a cura di), Rivoluzione visiva attraverso visioni rivoluzio- narie: alfabeti, cinema e letteratura in URSS, Dipartimento di Lingue e Culture Stra- niere Moderne, Torino 2018 (= "QuadRi. Quaderno di Ricognizioni”, VIII), pp. I46.
}

Il volume raccoglie relazioni presentate nel corso di due diversi e successivi congressi svoltisi a Torino nel novembre 2017 sulla scia delle celebrazioni del centenario della Rivoluzione d'Ottobre. Un primo gruppo di contributi (Sessione alfabetica), quantitativamente predominante e più omogeneo dal punto di vista tematico, è dedicato alla questione degli alfabeti nella discussione teorica e nella prassi politico-culturale della Repubblica russa, del Caucaso e dell'Asia centrale dopo il 1917. Il quadro di riferimento generale è opportunamente delineato nel saggio di V.S. Tomelleri, che ripercorre in modo sintetico il dibattito sulla questione delle nazionalità accesosi dopo la rivoluzione e mette a confronto le opinioni di esponenti bolscevichi di primo piano, spesso molto diverse fra loro e soggette a mutamenti nel corso della concreta esperienza di governo. Grazie alle sue molteplici competenze linguistiche il curatore riesce a fornire una articolata introduzione ai problemi politici, economici, psicologici generati fra le varie componenti del paese dalla ricerca di un sistema grafico adeguato alla nuova realtà, ma anche al dibattito appassionato fra gli specialisti, cui l'edificazione linguistica del nuovo regime offrì un'occasione storica per mettere alla prova metodi e teorie in territori vasti e differenziati.

La questione alfabetica fu uno dei tanti problemi resi ineludibili dopo la Rivoluzione dalla campagna per la liquidazione dell'analfabetismo; come sintetizza G. Schirru nel suo articolo, "l'obiettivo della campagna di istruzione era esplicitamente quello di alfabetizzare ciascuno dei cittadini sovietici nella sua lingua materna" e, data la diversità di condizioni di partenza delle varie lingue, fu necessario "porsi il compito di definire un insieme di varietà linguistiche di riferimento, fornire loro un sistema grafico stabile, un'adeguata elaborazione normativa e culturale” (p. 96). Di qui, in alcuni contesti, come il russo o l'armeno, il concretizzarsi di riforme ortografiche per cui da tempo erano maturati i presupposti e, per quanto riguardava i sistemi alfabetici, una serie di nuovi obiettivi: rompere con la tradizione imperiale russocentrica orientandosi verso sistemi grafici a base latina, offrire strumenti alfabetici adeguati alle lingue che ne erano ancora prive, ma anche superare l'eccessiva varietà dovuta a radicate tradizioni culturali, col rischio di suscitare reazioni di rigetto, soprattutto negli strati più acculturati delle diverse nazionalità. Gli articoli pubblicati nella raccolta descrivono alcune fasi di questo fermento riformatore: dai tentativi di unificazione dell'alfabeto (E. Simonato), alla scelta, in epoca post-sovietica, degli alfabeti per le lingue iraniche orientali del Tadžikistan (P. Ognibene), alla situazione armena (G. Schirru); vi si aggiungono il 
contributo di G. Selvelli sull'influenza del dibattito sovietico in Bulgaria nel periodo fra le due guerre, nel quale emergono i valori simbolici e ideologici dell'alfabeto, e di V.S. Tomelleri sulla tormentata storia del dizionario trilingue (osseto-russo-tedesco) di Vs.F. Miller e della sua ricezione in Occidente e nell'Unione Sovietica, soggetta da una parte e dall'altra a condizionamenti ideologici nonostante l'oggettiva importanza dell'opera.

Più ancora delle conclusioni dei singoli saggi, preziosi anche per i rinvii alla ormai ragguardevole bibliografia sull'argomento, l'interesse della raccolta risiede nella possibilità per il lettore di cogliere le analogie nell'evoluzione del dibattito sulle varie lingue dell' Unione Sovietica, pur nella specificità delle singole situazioni e nella diversità delle condizioni di partenza. Quasi ovunque la storia delle discussioni sulla scelta dell'alfabeto si trasformò in una lunga competizione fra latino e cirillico, nel corso della quale il valore attribuito all'uno o all'altro si modificava sotto la pressione di nuove circostanze, non solo politiche, e gli argomenti adottati a favore di una opzione potevano essere riutilizzati a favore di quella opposta. Merito degli autori, pur nella necessaria sintesi, è inoltre di aver fatto emergere la complessità del percorso che ha portato alla situazione attuale, mettendo in guardia da soluzioni semplicistiche e chiarendo come alcuni progetti, (ad esempio quello per la creazione di un alfabeto unificato su base latina, sostenuto da N.F. Jakovlev alla fine degli anni Venti: si veda il saggio di E. Simonato) fallirono per debolezza intrinseca, e non solo per le crescenti pressioni ideologiche.

La seconda parte del volume comprende due contributi dedicati al cinema e molto diversi fra loro. Il primo, di M. Tria, attraverso l'analisi di alcuni film russi degli ultimi anni e di documenti ufficiali di indirizzo, registra la presa di distanza ("salomonica") dell'attuale gruppo dirigente del Cremlino rispetto alla tradizionale esaltazione della rivoluzione del 1917, vista ormai come evento divisivo: un fenomeno rilevato da più parti durante le celebrazioni del centenario, e che trova qui una conferma convincente. Del tutto diverso è l'approccio nell'altro saggio, dove K. Ičin mette a confronto il film di animazione Potec (1992) di A. Fedulov con l'omonima pièce di A. Vvedenskij da cui è tratto, mostrando l'intima aderenza della realizzazione visuale al contenuto metafisico e alla poetica di Vvedenskij e, più in generale, degli oberjuty.

Nella sua prefazione il curatore M. Maurizio ricorda che uno dei due convegni confluiti nel libro aveva il titolo Strategia di sopravvivenza dopo il I9I7: quanto mai pertinente, in un volume prevalentemente dedicato alla cultura visiva, è quindi il saggio di N. A. Bogomolov, ricco di prezioso materiale d'archivio, sulla biografia di alcuni collaboratori, noti e meno noti, del giornale anarchico “Žizn”, uscito per soli due mesi nel 1918. Le loro vicende di esilio, di repressione o viceversa di una tranquilla sopravvivenza nel regime sovietico compongono una sorta di enciclopedia dei destini di un'intera generazione di letterati, la cui salvezza o annientamento furono spesso frutto del caso. 